\title{
Boletín
}

Criminológico

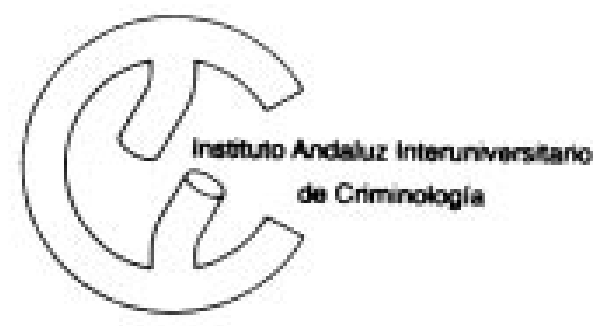

Directora: Elisa García España

Coordinadora: Susana García Ruiz

Publicado por la Sección de Málaga del IAIC

Edificio Institutos de Investigación, Universidad

de Málaga. Campus de Teatinos, 29071 MALAGA

Tel:(95) 2132325 - Fax:(95) 2132242

Depósitolegal:MA 857/1996ISSN:1137-2427

www.uma.es/estudios/propias/criminologia

Hace un año y medio en el Boletín Criminológico n ${ }^{\circ} 46$ presentábamos la memoria de actuaciones de la oficina del SAVA de Málaga en el primer aniversario de su constitución. En ella se resaltaba el número de usuarios, actuaciones y servicios prestados por esa oficina y se avanzaba una iniciativa pionera relativa a la puesta en marcha de un programa piloto de guardias de 24 horas los fines de semana y días festivos. Un año después dicho proyecto se ha hecho realidad. Con ello se advierte que las oficinas del SAVA no se limitan a atender las demandas que les llegan, sino que a partir de ellas toman iniciativas para prestar un mejor servicio y en una franja horaria más amplia.

En este boletín se ha querido recoger el primer año de experiencia de esta iniciativa, constatando la necesidad de cobertura asistencial a la víctima en la franja horaria no habitual de oficina y, por tanto, el éxito de la idea.

\section{PROGRAMA PILOTO DE
DEL SAVA DE MÁLAGA}

\section{Autora:}

María Victoria Rosas Lozano

En febrero de 2000 el Servicio de Atención a Víctimas de Delitos de Málaga puso en marcha el Servicio de Guardia de 24 horas los fines de semana y días festivos como experiencia piloto en Andalucía. Ninguna otra oficina de asistencia a víctimas del país presta funciones de guardia.

El proyecto surgió porque entendimos que existía una franja horaria en la que no había ningún recurso que prestara una ayuda adecuada a las víctimas, preocupándonos especialmente los casos graves en los que las mismas necesitan una intervención en crisis.

Ningún servicio de urgencia de los existentes (policial, sanitario, judicial...) ofrece a estas víctimas una ayuda especializada basada en criterios de inmediatez, empatía, apoyo personal y emocional, acompañamiento, asistencia jurídica, psicológica y social, búsqueda de los recursos necesarios, e incluso prestación económica de urgencia en algunos casos. Nuestro objetivo era paliar en la medida de lo posible los efectos traumáticos que derivan del delito, tratando de evitar la llamada victimación secundaria.

\section{Actuaciones del Servicio}

Desde que comenzó el servicio hasta final de año hemos cubierto un total de 48 fines de semana y 12 días festivos.

El número total de casos asistidos fue de 92. En 24 de ellos se hizo acto de presencia y 66 fueron atendidos telefónicamente (Figura $1)$.

En estas últimas atenciones el motivo de la consulta fue diverso, aunque destaca, en el $75 \%$ de los casos, la demanda de asesora-

\section{METODOLOGIA}

Los cuatro miembros del equipo técnico del SAVA cubren personalmente este servicio, de tal manera que durante cada turno la persona que está de guardia se encuentra localizable en todo momento a través de un teléfono móvil. Dependiendo de la gravedad del caso se atiende a la víctima telefónicamente, haciendo las gestiones oportunas sin necesidad de que el profesional se desplace o, si el asunto lo requiere, se acude al lugar donde se encuentre la víctima. De cualquier forma, la asistencia se hace siempre presencial en los casos de agresiones sexuales, malos tratos graves, y en los de desamparo absoluto de la víctima.

El horario de la Guardia es:

- Fines de semana: desde las 20:00 horas del viernes hasta las 9:00 horas del lunes.

- Días festivos: desde las 20:00 horas del día anterior al festivo hasta las 9:00 horas del siguiente.

\section{Boletín Criminológico $N^{\circ} 54$ Julio-Agosto 2001 Página 1}




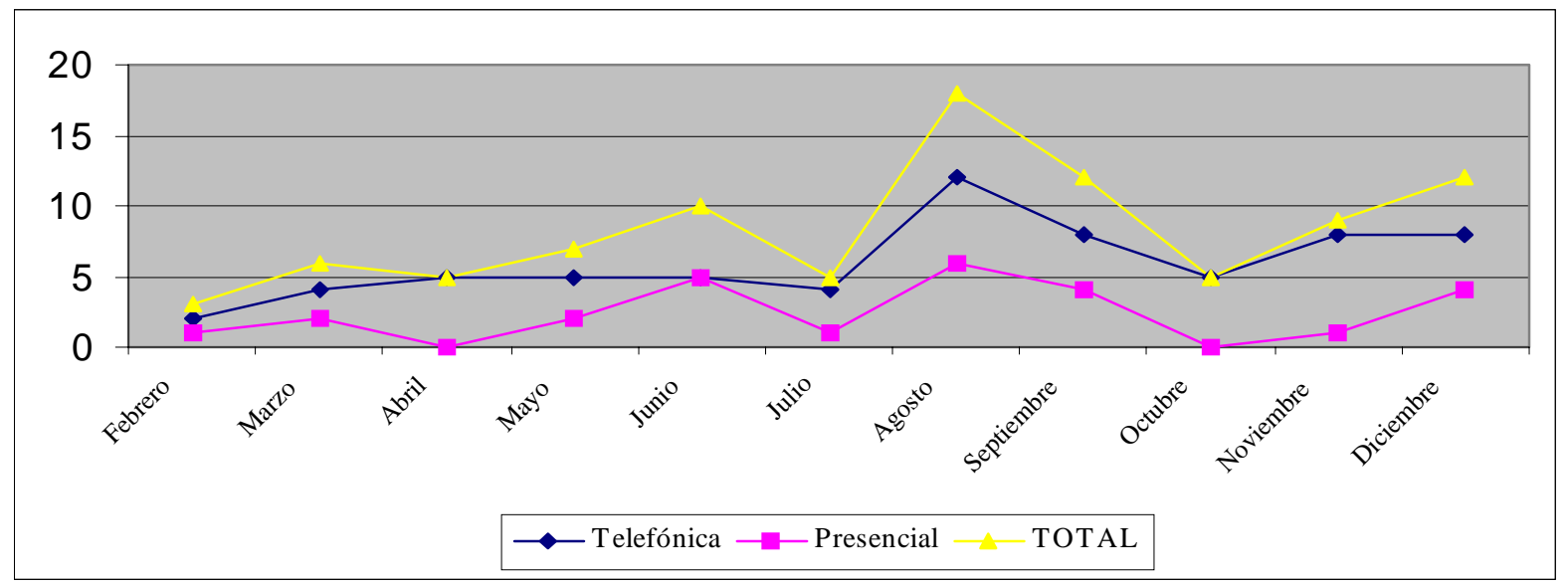

miento jurídico e información de recursos en temas de malos tratos y conflictos familiares.

De los actos presenciales hay que destacar la asistencia a 10 víctimas de malos tratos, 7 de agresiones sexuales (una de ellas sufrió también una detención ilegal) y otros 7 de delitos contra la propiedad.

Con relación a la franja horaria se puede observar en la Figura 2 , que el tramo de más demanda es el de las 20-24 horas que junto con la franja de 16-20 horas suponen los tramos horarios de mayor seguimiento del servicio de urgencia.

Otro dato a tener en cuenta es que en un $81,5 \%$ de los casos las atenciones fueron a mujeres.

La fuente de remisión más habitual es la Policía Nacional (25\%), seguida de los hospitales y centros de salud $(23,9 \%)$ y del juzgado de guardia $(21,7 \%)$.

El tipo de actuación practicada ha sido muy variada. No obstante lo más habitual ha sido hacer intervención en crisis, que en el caso de las asistencias presenciales ha consistido en la información de recursos y asesoramiento jurídico, acompañamientos, gestión de recursos, gestiones con la policía, hospitales y juzgado, derivación al SAVA, seguimiento de casos, etc.

Para hacer el análisis cualita- tivo de la calidad del servicio prestado, la necesidad del mismo y la duplicidad de recursos, veamos cuáles son las actuaciones que se vienen haciendo en los distintos casos y si existen o no otros recursos que cubran esas asistencias:

Guardias con asistencia telefónica

La mayoría de las llamadas recibidas están relacionadas con malos tratos y conflictos familiares. La asistencia normalmente consiste en asesoramiento jurídico, información sobre recursos, gestiones con entidades y derivación al SAVA en horario de oficina.

Si bien en ocasiones se producen consultas de cuestiones no urgentes, son numerosas las veces en las que la persona que llama se encuentra en un fuerte estado de ansiedad, llanto y angustia.

También por teléfono se hace intervención en crisis, se presta el apoyo emocional necesario y se realizan las gestiones y el seguimiento que el caso requiera.

Normalmente las llamadas que se producen de madrugada son casos de malos tratos, y proceden de hospitales o de la policía, o incluso de la propia víctima usuaria del SAVA.

A veces el asesoramiento es requerido por el propio médico o agente de policía, que necesita aclarar los pasos que deben seguir en relación principalmente con temas de agresiones sexuales y malos tratos: las dudas que se plantean están en torno a la aplicación del Procedimiento de Coordinación para atención a las mujeres víctimas de este tipo de delitos firmado al efecto en Sevilla el 26-111998 por el Consejero de la Presidencia, el delegado del Gobierno en Andalucía, la Consejera de Gobernación y Justicia, el Presidente del TSJA, el Consejero de Salud, el Consejero de Asuntos Sociales y el Presidente de la Federación Andaluza de Municipios y Provincias.

Actualmente sólo los casos de violencia de género (entre parejas) pueden encontrar otros recursos distintos al nuestro que cubran esta asistencia.

Guardias con asistencia presencial

Para desplazarnos al lugar donde se encuentra la víctima valoramos la gravedad del delito $\mathrm{y} / \mathrm{o}$ el estado de la víctima. A consecuencia del delito sufrido ésta se encuentra en una situación especialmente vulnerable, por lo que una asistencia adecuada a tiempo es decisiva para evitar mayores perjuicios. Una ayuda empática y receptiva palía el fuerte impacto psicológico que la victimización produce en cualquier persona. Una atención especializada produce un refuerzo del nivel afectivo y una

\section{Boletín Criminológico $N^{\circ} 54$ Julio-Agosto 2001 Página 2}


disminución del grado de ansiedad y de estrés.

La víctima es escuchada y se le comunica que el peligro ya ha pasado y que se encuentra a salvo, que la situación está controlada y que se la va a ayudar. Se le intenta adecuar el nivel del lenguaje, aclarando cualquier término técnico que pueda resultarle incomprensible. Se la acompaña a donde sea necesario (Juzgado de Guardia, centro de salud u hospitalario, domicilio, casa de acogida, etc.). Se procura evitar en ella actitudes de culpabilidad y se la hace partícipe en el autocontrol y normalización de la situación estresante, haciéndole conocer cuál es la situación real y el procedimiento que se va a seguir.

En definitiva, en todas las asistencias presenciales se realiza una intervención en crisis y un asesoramiento jurídico, $y$, a partir de ahí, las gestiones que resulten oportunas al caso.

La tipología delictiva que más se repite en este tipo de asistencias es la siguiente:

\section{$1^{\circ}$.- Agresiones sexuales}

Estas merecen especial relevancia por su frecuencia (casi un $27 \%$ del total) y gravedad. Después de la agresión sexual se produce en la víctima una crisis tan intensa que si no recibe una inmediata intervención afectiva, se puede agudizar el shock postraumá- tico. Además de las consecuencias clínicas agudas que se puedan plantear (lesiones genitales, embarazos, enfermedades de transmisión sexual, dolencias crónicas, etc.), las alteraciones psíquicas tras la violación se aprecian en éste como en ningún otro delito desde los primeros momentos.

Una pronta y adecuada intervención puede incidir de forma positiva en la fase aguda del denominado "síndrome del trauma de la violación", que tiene lugar inmediatamente después de la victimación y que puede durar unos días o unas pocas semanas. Esta fase tiene dos cuadros: el primero, que se manifiesta por sentimientos de miedo, ira y ansiedad demostrada por llantos, quejidos, sonrisas, inquietud y tensión; el segundo, por control de la conducta y enmascaramiento u ocultación de sentimientos. Un acompañamiento empático a la víctima en este momento es fundamental. Ningún recurso cubre esta asistencia. Sólo esta actuación ya justificaría desde nuestro punto de vista la existencia del Servicio de Guardia.

Superada la intervención en crisis, se ayuda a encajar el primer impacto de la victimación, dándose paso a la fase de pseudoadaptación y de integración, aunque en la mayoría de los casos no se logra una resolución satisfactoria y total de lo ocurrido, quedando una

\section{Figura 2: Franjas horarias}

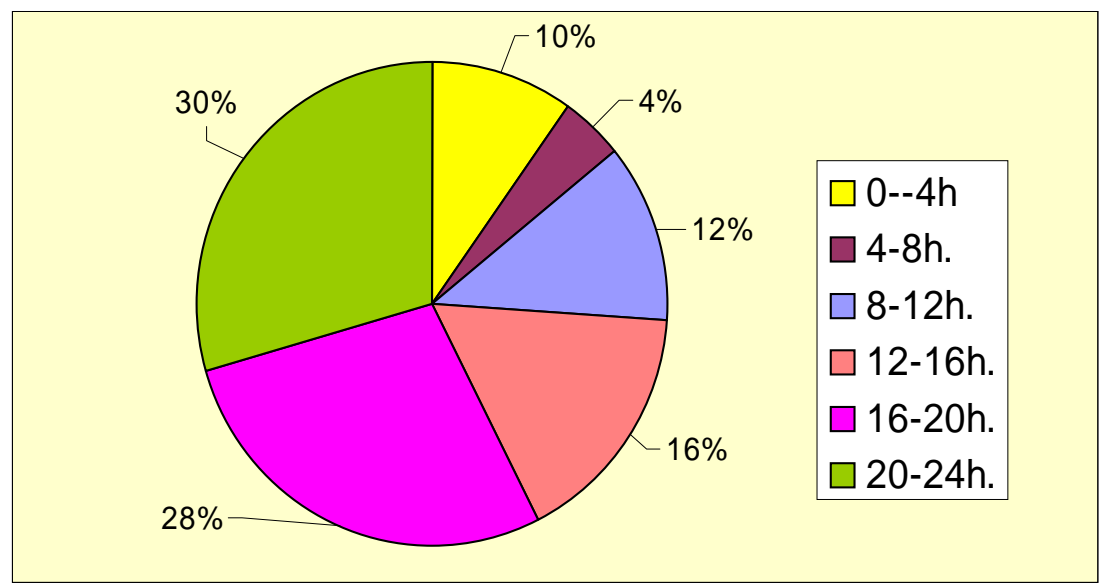

serie de secuelas psíquicas crónicas.

Todas las asistencias prestadas durante la guardia en estos casos han llevado consigo la apertura posterior de un expediente y el oportuno seguimiento tanto a nivel psicológico como jurídico desde el SAVA.

Por otra parte, nuestra presencia en el hospital en estos casos está haciendo que cada vez se sigan con mayor rigor todas las pautas previstas en el ya referido Procedimiento de Coordinación, de forma que se vela por la intimidad de la víctima llevándola a una sala distinta de la de espera, se recuerda al médico que ha de cumplimentar el parte de lesiones específico (creado al efecto para estos supuestos), se requiere la presencia de la policía en el propio centro médico para la toma de declaración, así como se hacen las gestiones oportunas con el Juez de Guardia a fin de que ordene al médico forense su traslado al hospital para que se practique a la víctima un único y conjunto reconocimiento. Conseguir todos estos objetivos no está resultando nada fácil ya que supone romper la dinámica acostumbrada.

\section{$2^{\circ}$.- Violencia doméstica}

Representan el 38.5\% de las asistencias prestadas.

Las mujeres, después de una situación de crisis (agresión reciente), presentan una alto nivel de ansiedad, miedo, dolor, humillación, vergüenza, angustia, culpabilidad, desesperanza, impotencia, vulnerabilidad, inseguridad, sentimiento de fracaso... Son sentimientos que desbordan a la mujer en el momento en que su pareja les agrede. Es el "síndrome de maltrato a la mujer" (SIMAM).

Cuando la víctima pide auxilio porque su pareja la maltrata, está pidiendo una ayuda concreta e inmediata y es el momento de inter-

\section{Boletín Criminológico $N^{o} 54 \quad$ Julio-Agosto 2001 Página 3}


venir. Una respuesta apropiada en el momento de crisis constituye la primera etapa en el largo proceso que conducirá a la ruptura del vínculo con el agresor.

Lo normal es que, cuando la mujer se decide a pedir ayuda, lleve años soportando malos tratos (a menudo acompañados de agresiones sexuales). Si no se interviene en ese preciso momento, haciéndole ver que hay solución a su problema y ofreciéndole los recursos que necesita, se pierde la oportunidad de que la víctima salga del círculo vicioso de violencia en el que se encuentra atrapada, porque a las pocas horas su pareja volverá a ella suplicándole el perdón con falsas promesas de haber cambiado, de que ya no la agredirá más, y conseguirá convencerla. Así hasta la próxima agresión, cerrando el llamado ciclo de violencia.

Con las víctimas de malos tratos es importante también realizar una tarea de prevención, para evitar que se reproduzcan las situaciones de agresión. En este sentido, hacemos hincapié con ellas en las medidas de seguridad que deben adoptar y en proporcionarles todos los teléfonos de urgencia.

Es posible pensar que en materia de malos tratos existen actualmente muchos recursos y que nuestro Servicio de Guardia para estos casos no sería necesario. Continuamente hemos de recordar que no somos un Servicio para mujeres maltratadas, y que la asistencia que prestamos a estas mujeres es sólo una parte de nuestro trabajo. Nosotros asistimos a "víctimas" de malos tratos, cualquiera que sea su sexo o edad y cualquiera que sea el maltratador (pareja, padres, hijos ...). Muchos de aquellos otros recursos sólo atienden a "mujeres víctimas de violencia de género", dejando sin cobertura el resto de los supuestos.

$3^{\circ}$.- Delitos contra la propiedad
Suponen otro $27 \%$ de los casos asistidos.

Cualquier persona víctima de un robo siente una gran frustración ante el hecho, pero si además el robo va acompañado de violencia o intimidación, las consecuencias y posteriores secuelas físicas y/o psicológicas son aún más graves.

No es lo mismo que roben a una persona joven o de mediana edad, sana y con recursos, a que lo hagan a una persona mayor, desvalida, extranjera o sin recursos. A veces estas circunstancias aparecen unidas en la misma víctima, con lo que la situación se complica bastante.

En este sentido, cinco de las siete asistencias prestadas en Comisaría por delitos contra la propiedad han sido precisamente a víctimas extranjeras, objetivo muy apetecible por los delincuentes. En estos casos, a las consecuencias normales de la comisión del delito se añade el problema del desconocimiento del idioma y de la falta de recursos de la víctima en el país (documentación, dinero, amigos o familiares que puedan ayudarle, domicilio, etc.). La víctima se encuentra totalmente desamparada, desorientada, sin saber qué hacer ni a dónde ir. Coincide además que, siendo festivo, su consulado o embajada están cerrados. La Policía, aparte de recogerle la denuncia, no puede hacer nada más. Sólo habría una solución para el alojamiento y la comida gratuita: el albergue municipal, y este es un lugar poco idóneo para según que casos. Ante el bloqueo de la situación, la Policía recurre al SAVA que, además de prestarle apoyo personal y emocional, asesoramiento adecuado, acompañamiento a donde proceda, la puesta en contacto con sus familiares, etc., le busca un alojamiento adecuado y, en caso necesario, sufraga los gastos imprescindibles para el alojamiento, manutención o transporte.

Tampoco en estos casos existe ningún otro recurso o servicio que cubra este tipo de asistencias.

\section{Valoración}

El nivel de agradecimiento de las víctimas a las que asistimos y acompañamos en las guardias es todavía superior al que muestran las que asistimos durante las jornadas normales de oficina, que ya es alto. El sentirse tan desamparadas, el encontrarse solas en un momento tan crítico, el no poder acudir a ningún recurso especializado por ser festivo y estar todo cerrado, les hace agradecer mucho más nuestra asistencia.

El SAVA ve cumplido pues, a través de este Servicio, su objetivo principal, que es la asistencia a las víctimas.

\section{PREMIO CONSTANCIO BERNALDO DE QUIRÓS}

- La sección de Cádiz del IAIC convoca la primera edición del premio Constancio Bernaldo de Quirós con la finalidad de galardonar el mejor trabajo de investigación de carácter criminológico.

- Podrán concurrir al certamen los alumnos que hayan cursado estudios en criminología en el curso académico 2000/2001 en alguna Universidad española y aquellos que se encuentren en posesión del título académico de Criminología que expidan dichas universidades.

- El plazo finaliza el 15 de Enero del 2002. Para mayor información puede ponerse en contacto con la sección de Cádiz a través de: Teléfono 9560307043

\section{Boletín Criminológico $N^{o} 54 \quad$ Julio-Agosto 2001 Página 4}

